\title{
A Barth-Lefschetz theorem for toric varieties
}

\author{
Jörg Zintl
}

Dedicated to the memory of Michael Schneider

1991 AMS Subject Classification: 14 F 25 (14 M 25, 14 M 07)

By the well known theorem of Barth-Lefschetz for complex projective space $Y=\mathbb{P}^{r}$ one has

$$
H^{q}(Y, X ; \mathbb{C})=0 \quad \text { for } q \leq 2 n-r+1
$$

for any $n$-dimensional submanifold $X \subseteq Y$. This was proved first by Barth [B] generalizing a theorem of Lefschetz on hypersurfaces $X$. Using Le Potier's vanishing theorem [LP] a new proof was given in a joint paper with Schneider [SZ]. That article contains also references to further proofs of the theorem. Using the same arguments as in [SZ, Debarre [D] was able to show that the theorem of Barth-Lefschetz holds for abelian varieties $Y$ as well, assuming that the normal bundle of $X$ in $Y$ is ample."

The idea behind this paper was to study the more general situation of a submanifold $X$ in a smooth projective toric variety $Y$. Giving up $\mathbb{P}^{r}$ as ambient space, which is very special indeed, one needs some additional assumptions on the submanifold $X$. One requirement is that the normal bundle of $X$ in $Y$ is ample, which is always true if $Y=\mathbb{P}^{r}$. Besides that, the submanifold should meet the boundary of $Y$ transversally, which again can be achieved in $\mathbb{P}^{r}$ using the homogeneity of projective space.

\footnotetext{
${ }^{1}$ In fact, this is a special case of a result on submanifolds of projective homogeneous manifolds which has been proved earlier by Sommese [So] using different methods.
} 
For an arbitrary such submanifold $X$ in $Y$ one cannot expect that the result of Barth-Lefschetz still holds. In theorem 16 a precise toric characterization is given in which cases it does. The main theorem 10 settles the general case. It gives a formula for all Hodge numbers $h^{p, q}(X)$ for any such submanifold $X$ in the Barth-Lefschetz range $p+q \leq 2 n-r$.

Lemma 1 Let $0 \rightarrow F \rightarrow E_{0} \rightarrow \ldots \rightarrow E_{k} \rightarrow 0$ be an exact sequence of sheaves on a scheme $Z$. Let $q \geq 0$ and assume

$$
H^{q-i}\left(Z, E_{i}\right)=H^{q-i-1}\left(Z, E_{i}\right)=0 \quad \text { for } 0 \leq i \leq k-1 .
$$

Then

$$
H^{q}(Z, F) \cong H^{q-k}\left(Z, E_{k}\right)
$$

Proof. By induction on $k$ and cutting the sequence into two pieces.

Let $Y$ denote a smooth projective toric variety of dimension $r>0$, given by a fan $\Delta$ in some lattice $N \cong \mathbb{Z}^{r}$. The dual lattice to $N$ will be called $M$. Each cone $\sigma \in \Delta$ corresponds to precisely one orbit of the action of $T_{N}=\left(\mathbb{C}^{*}\right)^{r}$ on $Y$. The closure of this orbit is denoted by $V(\sigma)$. It is again a smooth projective toric subvariety of codimension equal to the dimension of $\sigma$. Put

$$
\Delta(i):=\{\sigma \in \Delta: \operatorname{dim} \sigma=i\} .
$$

For each $0 \leq i \leq r$ the set $\Delta(i)$ is finite because $Y$ is compact. Since $Y$ is smooth, Ishida's complex of degree $p$

$$
0 \rightarrow \Omega_{Y}^{p} \rightarrow \mathcal{K}^{0}(Y ; p) \rightarrow \ldots \rightarrow \mathcal{K}^{p}(Y ; p) \rightarrow 0
$$

is exact for $p=0, \ldots, r$, where

$$
\mathcal{K}^{j}(Y ; p)=\bigoplus_{\sigma \in \Delta(j)} \mathcal{O}_{V(\sigma)} \otimes_{\mathbb{Z}} \bigwedge^{p-j}\left(M \cap \sigma^{\perp}\right)
$$

Note that $M \cap \sigma^{\perp}$ is a free $\mathbb{Z}$-module of rank $r-j$.

Let $X$ be a submanifold of $Y$ of dimension $n$ with ample normal bundle $\mathcal{N}_{X / Y}$. We assume $X$ to be in general position. This is made precise in the following definition. 
Definition 2 We say that $X \subset Y$ is $\Delta$-transversal if for all $\sigma \in \Delta(k)$ with $1 \leq k \leq n$ the intersection of $X$ with $V(\sigma)$ is either empty or transversal, and if it is empty for $k>n$.

From now on we will assume that $X$ is $\Delta$-transversal. In particular this implies that $X \cap V(\sigma)$ is smooth, if it is not empty, and

$$
\mathcal{N}_{X / Y} \mid X \cap V(\sigma) \cong \mathcal{N}_{X \cap V(\sigma) / V(\sigma)}
$$

is ample with $\operatorname{rk} \mathcal{N}_{X / Y} \mid X \cap V(\sigma)=\operatorname{rk} \mathcal{N}_{X \cap V(\sigma) / V(\sigma)}$.

We will need the following vanishing theorem due to Le Potier.

Theorem 3 (Le Potier) Let $Z$ be a smooth projective manifold of dimension $m$ and $E$ an ample vector bundle of rank $s$ on $Z$. Then

$$
H^{q}\left(Z, \Omega_{Z}^{p} \otimes E^{*}\right)=0 \quad \text { for } p+q \leq m-s .
$$

In particular by what we said above, for all $\sigma \in \Delta(i)$ one has

$$
H^{q}\left(X \cap V(\sigma), \mathcal{N}_{X \cap V(\sigma) / V(\sigma)}^{*}\right)=0 \quad \text { for } q \leq 2 n-r-i .
$$

We will use this to prove a vanishing theorem for symmetric powers of the conormal bundle $\mathcal{N}_{X / Y}^{*}$.

Theorem 4 Let $X$ be a $\Delta$-transversal submanifold of $Y$ with ample normal bundle $\mathcal{N}_{X / Y}$. Then for all $k \geq 1$

$$
H^{q}\left(X, S^{k} \mathcal{N}_{X / Y}^{*}\right)=0 \text { for } q \leq 2 n-r .
$$

Note that if $X$ is a hypersurface in $Y$ then the theorem is true even without any condition on the position of $X$. This follows immediately from Kodaira's vanishing theorem.

Proof. Since $S^{k+1} \mathcal{N}_{X / Y}^{*}$ is a direct summand of $S^{k} \mathcal{N}_{X / Y}^{*} \otimes \mathcal{N}_{X / Y}^{*}$ it suffices to show

$$
H^{q}\left(X, S^{k} \mathcal{N}_{X / Y}^{*} \otimes \mathcal{N}_{X / Y}^{*}\right)=0 \quad \text { for } k \geq 0 \quad \text { and } q \leq 2 n-r .
$$


If $k=0$ this is just Le Potier's theorem. We proceed by induction on $k$. Take symmetric powers of the conormal seqence

$$
0 \rightarrow \mathcal{N}_{X / Y}^{*} \rightarrow \Omega_{Y}^{1} \mid X \rightarrow \Omega_{X}^{1} \rightarrow 0
$$

to get

$$
0 \rightarrow S^{k} \mathcal{N}_{X / Y}^{*} \rightarrow S^{k-1} \mathcal{N}_{X / Y}^{*} \otimes \Omega_{Y}^{1}\left|X \rightarrow \ldots \rightarrow \Omega_{Y}^{k}\right| X \rightarrow \Omega_{X}^{k} \rightarrow 0 .
$$

Tensoring this with $\mathcal{N}_{X / Y}^{*}$ gives

$$
0 \rightarrow S^{k} \mathcal{N}_{X / Y}^{*} \otimes \mathcal{N}_{X / Y}^{*} \rightarrow E_{1} \rightarrow \ldots \rightarrow E_{k} \rightarrow \Omega_{X}^{k} \otimes \mathcal{N}_{X / Y}^{*} \rightarrow 0
$$

where

$$
E_{i}=S^{k-i} \mathcal{N}_{X / Y}^{*} \otimes \Omega_{Y}^{i} \mid X \otimes \mathcal{N}_{X / Y}^{*} \quad \text { for } 1 \leq i \leq k .
$$

It suffices to prove

$$
H^{q-i+1}\left(X, E_{i}\right)=H^{q-i}\left(X, E_{i}\right)=0 \quad \text { for } 1 \leq i \leq k
$$

and $q \leq 2 n-r$, because then by lemma 1

$$
H^{q}\left(X, S^{k} \mathcal{N}_{X / Y}^{*} \otimes \mathcal{N}_{X / Y}^{*}\right) \cong H^{q-k}\left(X, \Omega_{X}^{k} \otimes \mathcal{N}_{X / Y}^{*}\right)
$$

and we are reduced to Le Potier's theorem.

So fix an integer $1 \leq i \leq k$. We may assume $i \leq r$ since otherwise $E_{i}=0$ and we are done. By tensoring Ishida's complex of degree $i$ with $S^{k-i} \mathcal{N}_{X / Y}^{*} \otimes \mathcal{N}_{X / Y}^{*}$ we obtain

$$
\begin{aligned}
0 \rightarrow & E_{i} \rightarrow \mathcal{K}^{0}(Y ; i) \otimes S^{k-i} \mathcal{N}_{X / Y}^{*} \otimes \mathcal{N}_{X / Y}^{*} \rightarrow \ldots \\
& \ldots \rightarrow \mathcal{K}^{i}(Y ; i) \otimes S^{k-i} \mathcal{N}_{X / Y}^{*} \otimes \mathcal{N}_{X / Y}^{*} \rightarrow 0
\end{aligned}
$$

If $0 \leq \nu \leq i-1$ and $X \cap V(\sigma) \neq \emptyset$ for $\sigma \in \Delta(\nu)$, then $\mathcal{N}_{X / Y}^{*} \mid X \cap V(\sigma) \cong$ $\mathcal{N}_{X \cap V(\sigma) / V(\sigma)}^{*}$ as mentioned above, and one has

$$
H^{q}\left(X \cap V(\sigma), S^{k-i} \mathcal{N}_{X \cap V(\sigma) / V(\sigma)}^{*} \otimes \mathcal{N}_{X \cap V(\sigma) / V(\sigma)}^{*}\right)=0
$$

for $q \leq 2 n-r-\nu$ by induction on $k$. Hence

$$
H^{q}\left(X, \mathcal{K}^{\nu}(Y ; i) \otimes S^{k-i} \mathcal{N}_{X / Y}^{*} \otimes \mathcal{N}_{X / Y}^{*}\right)=0
$$


for $0 \leq \nu \leq i-1$ and $q \leq 2 n-r-\nu$. This implies by lemma 1

$$
H^{q}\left(X, E_{i}\right) \cong H^{q-i}\left(X, \mathcal{K}^{i}(Y ; i) \otimes S^{k-i} \mathcal{N}_{X / Y}^{*} \otimes \mathcal{N}_{X / Y}^{*}\right)
$$

for $q \leq 2 n-r$. Again by induction on $k$ this vanishes as we have $q-i \leq$ $2 n-r-i$.

As in SZ we will compare the cohomology groups $H^{k}(Y, \mathbb{C})$ and $H^{k}(X, \mathbb{C})$ using Hodge decomposition and factoring the natural map via

$$
H^{i}\left(Y, \Omega_{Y}^{j}\right) \rightarrow H^{i}\left(X, \Omega_{Y}^{j} \mid X\right) \rightarrow H^{i}\left(X, \Omega_{X}^{j}\right)
$$

Proposition 5 Let $X \subset Y$ be as above. The natural map

$$
H^{i}\left(X, \Omega_{Y}^{j} \mid X\right) \rightarrow H^{i}\left(X, \Omega_{X}^{j}\right)
$$

is surjective if $i+j \leq 2 n-r$ and injective if $i+j \leq 2 n-r+1$.

Proof. Consider the $j$-th symmetric power of the conormal sequence

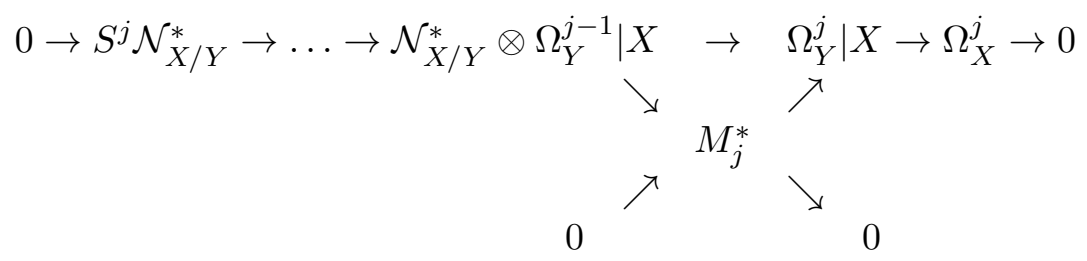

and cut it into two pieces. We have to prove $H^{i}\left(M_{j}^{*}\right)=0$ for $i+j \leq$ $2 n-r+1$. Look at Ishida's complex of degree $\nu$ tensored with $S^{j-\nu} \mathcal{N}_{X / Y}^{*}$ for $1 \leq \nu \leq j-1$.

$$
\begin{gathered}
0 \rightarrow \Omega_{Y}^{\nu} \mid X \otimes S^{j-\nu} \mathcal{N}_{X / Y}^{*} \rightarrow \mathcal{K}^{0}(Y ; \nu) \otimes S^{j-\nu} \mathcal{N}_{X / Y}^{*} \rightarrow \ldots \\
\ldots \rightarrow \mathcal{K}^{\nu}(Y ; \nu) \otimes S^{j-\nu} \mathcal{N}_{X / Y}^{*} \rightarrow 0
\end{gathered}
$$

As in the proof of theorem 4 we use the isomorphism $\mathcal{N}_{X / Y}^{*} \mid X \cap V(\sigma) \cong$ $\mathcal{N}_{X \cap V(\sigma) / V(\sigma)}^{*}$ for $\sigma \in \Delta(\mu)$ if $X \cap V(\sigma) \neq \emptyset$. We obtain

$$
H^{q}\left(X, \mathcal{K}^{\mu}(Y ; \nu) \otimes S^{j-\nu} \mathcal{N}_{X / Y}^{*}\right)=0 \quad \text { for } q \leq 2 n-r-\mu
$$


and $0 \leq \mu \leq \nu-1$ by theorem 4 . By the same theorem and by lemma 1 we have

$$
H^{q}\left(\Omega_{Y}^{\nu} \mid X \otimes S^{j-\nu} \mathcal{N}_{X / Y}^{*}\right) \cong H^{q-\nu}\left(X, \mathcal{K}^{\nu}(Y ; \nu) \otimes S^{j-\nu} \mathcal{N}_{X / Y}^{*}\right)=0
$$

if $q \leq 2 n-r$. Now we apply this and lemma 1 to the long exact sequence containing $M_{j}^{*}$ above. We get

$$
H^{i+j-1}\left(X, S^{j} \mathcal{N}_{X / Y}^{*}\right) \cong H^{i}\left(X, M_{j}^{*}\right)
$$

for $i+j-1 \leq 2 n-r$. Since the left hand side vanishes by theorem 4 , we finally obtain $H^{i}\left(X, M_{j}^{*}\right)=0$ for $i+j \leq 2 n-r+1$ as desired.

Before we can proceed we need to show the following general fact.

Proposition 6 Let $Z$ be a smooth projective variety and let $W \subset Z$ be a submanifold with ample normal bundle $\mathcal{N}_{W / Z}$. Let $D_{1}, \ldots, D_{s}$ be hypersurfaces on $Z$ such that $D_{i} \cap W=\emptyset$ for all $i=1, \ldots, s$. Assume that there are integers $a_{1}, \ldots, a_{s} \in \mathbb{Z}$ such that $\sum_{i=1}^{s} a_{i} D_{i}$ is linearly equivalent to 0 . Then $a_{i}=0$ for all $i=1, \ldots, s$.

Proof. Assume that the claim does not hold. We denote by $D_{0}$ the effective divisor $\sum a_{i} D_{i}$ where the sum runs over all $a_{i}>0$, and by $D_{\infty}$ the effective divisor $\sum\left|a_{i}\right| D_{i}$ where $a_{i}<0$. Since by assumption $D_{0}-D_{\infty}$ is linearly equivalent to 0 , there exists a global meromorphic function $g$ on $Z$, such that

$$
(g)=D_{0}-D_{\infty}
$$

Since $g$ has neither poles nor zeroes when restricted to $W$, its restriction has to be equal to a constant $c$, say. Hence the function $g-c$ vanishes on $W$, and it is defined near $W$. Since it is not identically 0 , there is some integer $k$ such that $g-c$ belongs to $\mathcal{I}_{W}^{k}$ but $g-c$ does not belong to $\mathcal{I}_{W}^{k+1}$, where $\mathcal{I}_{W}$ denotes the ideal sheaf of $W$ in $Z$. Hence $g-c$ provides a non-zero section of $\mathcal{I}_{W}^{k} / \mathcal{I}_{W}^{k+1}=S^{k} \mathcal{N}_{W / Z}^{*}$, which contradicts the ampleness of $\mathcal{N}_{W / Z}$.

Corollary 7 Let $X \subset Y$ be as before. Let $s$ be the number of $\sigma \in \Delta(1)$ such that $V(\sigma) \cap X=\emptyset$. Then there is a short exact sequence

$$
0 \rightarrow \Omega_{Y}^{1} \mid X \rightarrow \bigoplus_{\substack{\sigma \in \Delta(1) \\ V(\sigma) \cap X \neq \emptyset}} \mathcal{O}_{X}(-V(\sigma) \cap X) \rightarrow \mathcal{O}_{X}^{\delta(1)-r-s} \rightarrow 0 .
$$


Proof. By $\mathrm{BC}$ there is an exact generalized Euler sequence

$$
0 \rightarrow \Omega_{Y}^{1} \rightarrow \bigoplus_{\sigma \in \Delta(1)} \mathcal{O}_{Y}(-V(\sigma)) \rightarrow \mathcal{O}_{Y}^{\delta(1)-r} \rightarrow 0
$$

By construction the second map factors as

$$
\bigoplus_{\sigma \in \Delta(1)} \mathcal{O}_{Y}(-V(\sigma)) \rightarrow \bigoplus_{\sigma \in \Delta(1)} \mathcal{O}_{Y} \rightarrow \bigoplus_{i=1}^{\delta(1)-r} \mathcal{O}_{Y}
$$

where the first map is injective, and the second map is obtained from the standard exact sequence

$$
0 \rightarrow M \rightarrow \bigoplus_{\sigma \in \Delta(1)} \mathbb{Z} V(\sigma) \rightarrow \operatorname{Pic}(Y) \rightarrow 0
$$

by tensoring with $\mathcal{O}_{Y}$. Here $\bigoplus \mathbb{Z} V(\sigma)$ denotes the free group generated by the boundary divisors $V(\sigma)$, and we use $\operatorname{Pic}(Y) \cong \mathbb{Z}^{\delta(1)-r}$. Now proposition 6 implies that the map

$$
\bigoplus_{\substack{\sigma \in \Delta(1) \\ V(\sigma) \cap X=\emptyset}} \mathbb{Z} V(\sigma) \rightarrow \operatorname{Pic}(Y)
$$

is injective. This remains true when we tensor with $\mathcal{O}_{X}$. Hence the gereralized Euler sequence restricted to $X$ splits off $s$ copies of $\mathcal{O}_{X}$, one for each $\sigma \in \Delta(1)$ with $V(\sigma) \cap X=\emptyset$, and thus reduces to a short exact sequence

$$
0 \rightarrow \Omega_{Y}^{1} \mid X \rightarrow \bigoplus_{\substack{\sigma \in \Delta(1) \\ V(\sigma) \cap X \neq \emptyset}} \mathcal{O}_{X}(-V(\sigma) \cap X) \rightarrow \mathcal{O}_{X}^{\delta(1)-r-s} \rightarrow 0
$$

as claimed.

Using this observation, we can begin to determine Hodge numbers of $X \subset Y$.

Proposition 8 Let $X \subset Y$ be as above and connected. Then for the Hodge numbers of $X$ holds

$$
h^{i, 0}(X)=h^{0, i}(X)=0
$$

for $1 \leq i \leq 2 n-r$. 
Proof. Note that for $i \geq 1$ we have a natural inclusion

$$
\Omega_{Y}^{i} \hookrightarrow \Omega_{Y}^{i-1} \otimes \Omega_{Y}^{1}
$$

as a direct summand. Tensoring the exact sequence of corollary 7 with $\Omega_{Y}^{i-1} \mid X$ we get an injective map

$$
\Omega_{Y}^{i-1}\left|X \otimes \Omega_{Y}^{1}\right| X \hookrightarrow \bigoplus_{\substack{\sigma \in \Delta(1) \\ V(\sigma) \cap X \neq \emptyset}} \Omega_{Y}^{i-1} \mid X \otimes \mathcal{O}_{X}(-V(\sigma) \cap X)
$$

Hence for each $\tau \in \Delta(1)$ there is a chain of injections

$$
\begin{aligned}
& H^{0}\left(X, \Omega_{Y}^{i} \mid X \otimes \mathcal{O}_{X}(-V(\tau) \cap X)\right) \rightarrow H^{0}\left(X, \Omega_{Y}^{i} \mid X\right) \rightarrow \\
\rightarrow & H^{0}\left(X, \Omega_{Y}^{i-1}\left|X \otimes \Omega_{Y}^{1}\right| X\right) \rightarrow \bigoplus_{\substack{\sigma \in \Delta(1) \\
V(\sigma) \cap X \neq \emptyset}} H^{0}\left(X, \Omega_{Y}^{i-1} \mid X \otimes \mathcal{O}_{X}(-V(\sigma) \cap X)\right)
\end{aligned}
$$

If $i=1$ then the sum of cohomology groups on the right hand side vanishes, and so does the whole chain. Inductively this shows

$$
H^{0}\left(X, \Omega_{Y}^{i} \mid X\right)=0
$$

for all $i \geq 1$. Because of proposition 5 and Hodge symmetry the claim follows.

Definition 9 Let $\delta(i)$ denote the number of elements in $\Delta(i)$. We write $\delta_{X, \sigma}$ for the number of connected components of $X \cap V(\sigma)$ for $\sigma \in \Delta$. Put

$$
\delta_{X}(i):=\sum_{\sigma \in \Delta(i)} \delta_{X, \sigma}
$$

Using this notation we can state our main theorem.

Theorem 10 Let $Y$ be a smooth projective toric variety of dimension $r$ and let $X$ be a $n$-dimensional, connected and $\Delta$-transversal submanifold of $Y$ with ample normal bundle. Then for $i+j \leq 2 n-r$ the following formula holds for the Hodge numbers of $X$.

$$
h^{i}\left(X, \Omega_{X}^{j}\right)= \begin{cases}\sum_{k=0}^{j}(-1)^{j-k}\left(\begin{array}{c}
r-k \\
j-k
\end{array}\right) \delta_{X}(k) & \text { if } i=j \\
0 & \text { if } i \neq j .\end{cases}
$$


Proof. If $i=j=0$ this is clear. If either $i=0$ or $j=0$ vanishing of the cohomology was proved in proposition 8. By proposition 月 it is sufficient to determine the dimensions of $H^{i}\left(X, \Omega_{Y}^{j} \mid X\right)$ for $i+j \leq 2 n-r$. Let us assume $i>j$ first. Since proposition 8 implies $H^{q}\left(X, \mathcal{K}^{\nu}(Y ; j) \mid X\right)=0$ for $1 \leq q \leq 2 n-r-\nu$ we have by lemma 1 applied to Ishida's complex of degree $j$ and restricted to $X$

$$
H^{i}\left(X, \Omega_{Y}^{j} \mid X\right) \cong H^{i-j}\left(X, \mathcal{K}^{j}(Y ; j) \mid X\right)=0 .
$$

Because of proposition 5 and Hodge symmetry we have

$$
H^{i}\left(X, \Omega_{Y}^{j} \mid X\right) \cong H^{i}\left(X, \Omega_{X}^{j}\right) \cong H^{j}\left(X, \Omega_{X}^{i}\right) \cong H^{j}\left(X, \Omega_{Y}^{i} \mid X\right)
$$

for $i+j \leq 2 n-r$. Hence the proposition is true for $i<j$ as well.

It remains to look at the case $i=j$ and $i \neq 0$. In particular we have then $i \leq \frac{2 n-r}{2}$. If $i=1$ the claim follows from the short exact sequence

$$
0 \rightarrow \Omega_{Y}^{1}\left|X \rightarrow \mathcal{K}^{0}(Y ; 1)\right| X \rightarrow \mathcal{K}^{1}(Y ; 1) \mid X \rightarrow 0
$$

and proposition 8. Otherwise consider Ishida's complex of degree $i \geq 2$ cut into pieces.

$$
\begin{gathered}
0 \rightarrow \Omega_{Y}^{i} \rightarrow \mathcal{K}^{0}(Y ; i) \rightarrow \mathcal{L}^{1} \rightarrow 0 \\
0 \rightarrow \mathcal{L}^{1} \rightarrow \mathcal{K}^{1}(Y ; i) \rightarrow \mathcal{L}^{2} \rightarrow 0 \\
\vdots \\
0 \rightarrow \mathcal{L}^{i-1} \rightarrow \mathcal{K}^{i-1}(Y ; i) \rightarrow \mathcal{K}^{i}(Y ; i) \rightarrow 0
\end{gathered}
$$

The $\mathcal{L}^{\nu}$ for $1 \leq \nu \leq i-1$ are inductively defined by this short exact sequences. We restrict them to $X$. Since $H^{\mu}\left(X, \mathcal{K}^{\nu}(Y ; i) \mid X\right)=0$ for $1 \leq \mu \leq 2 n-r-\nu$ we have

$$
H^{q}\left(X, \Omega_{Y}^{i} \mid X\right) \cong H^{q-1}\left(X, \mathcal{L}^{1} \mid X\right) \cong \ldots \cong H^{q-i+1}\left(X, \mathcal{L}^{i-1} \mid X\right)
$$

if $2 n-r \geq q \geq i$. This vanishes if $q \neq i$ as we have seen just before. In other words

$$
H^{q}\left(X, \mathcal{L}^{p} \mid X\right)=0 \quad \text { if } 1 \leq p \leq i-1,1 \leq q \leq 2 n-r-p \quad \text { and } p+q \neq i .
$$

If $q=i$ we get

$$
H^{i}\left(X, \Omega_{Y}^{i} \mid X\right) \cong H^{i-1}\left(X, \mathcal{L}^{1} \mid X\right) \cong \ldots \cong H^{1}\left(X, \mathcal{L}^{i-1} \mid X\right) .
$$


We obtain also an exact sequence

$$
\begin{aligned}
0 \rightarrow H^{0}\left(X, \mathcal{L}^{i-1} \mid X\right) \rightarrow H^{0}\left(X, \mathcal{K}^{i-1}(Y ; i) \mid X\right) \rightarrow \\
\quad \rightarrow H^{0}\left(X, \mathcal{K}^{i}(Y ; i) \mid X\right) \rightarrow H^{1}\left(X, \mathcal{L}^{i-1} \mid X\right) \rightarrow 0
\end{aligned}
$$

as well as

$$
\begin{gathered}
0 \rightarrow H^{0}\left(X, \mathcal{L}^{i-2} \mid X\right) \rightarrow H^{0}\left(X, \mathcal{K}^{i-2}(Y ; i) \mid X\right) \rightarrow H^{0}\left(X, \mathcal{L}^{i-1} \mid X\right) \rightarrow 0 \\
\vdots \\
0 \rightarrow H^{0}\left(X, \mathcal{L}^{1} \mid X\right) \rightarrow H^{0}\left(X, \mathcal{K}^{1}(Y ; i) \mid X\right) \rightarrow H^{0}\left(X, \mathcal{L}^{2} \mid X\right) \rightarrow 0 \\
0 \rightarrow H^{0}\left(X, \Omega_{Y}^{i} \mid X\right) \rightarrow H^{0}\left(X, \mathcal{K}^{0}(Y ; i) \mid X\right) \rightarrow H^{0}\left(X, \mathcal{L}^{1} \mid X\right) \rightarrow 0
\end{gathered}
$$

By proposition 8 the last exact sequence simply says

$$
H^{0}\left(X, \mathcal{K}^{0}(Y ; i) \mid X\right) \cong H^{0}\left(X, \mathcal{L}^{1} \mid X\right) .
$$

Taking everything together we get a long exact sequence

$$
\begin{aligned}
0 \rightarrow H^{0}\left(X, \mathcal{K}^{0}(Y ; i) \mid X\right) \rightarrow H^{0}\left(X, \mathcal{K}^{1}(Y ; i) \mid X\right) \rightarrow \ldots \\
\ldots \rightarrow H^{0}\left(X, \mathcal{K}^{i}(Y ; i) \mid X\right) \rightarrow H^{i}\left(X, \Omega_{Y}^{i} \mid X\right) \rightarrow 0 .
\end{aligned}
$$

So adding up all contributions finally gives

$$
\begin{aligned}
h^{i}\left(X, \Omega_{Y}^{i} \mid X\right) & =\sum_{\nu=0}^{i}(-1)^{i-\nu} h^{0}\left(X, \mathcal{K}^{\nu}(Y ; i) \mid X\right) \\
& =\sum_{\nu=0}^{i}(-1)^{i-\nu}\left(\begin{array}{c}
r-\nu \\
i-\nu
\end{array}\right) \delta_{X}(\nu) .
\end{aligned}
$$

Remark 11 The vanishing statement of the theorem above can be proved without referring to the toric nature of $Y$. We only need to know that $H^{i}\left(Y, \Omega_{Y}^{j}\right)=0$ for $i \neq j$, and that the normal bundle of $X$ in $Y$ is ample. This fact, and the proof of the lemma below, were kindly pointed out to me by Claire Voisin.

Let $j: X \rightarrow Y$ denote the inclusion. Then there is a commutative diagram

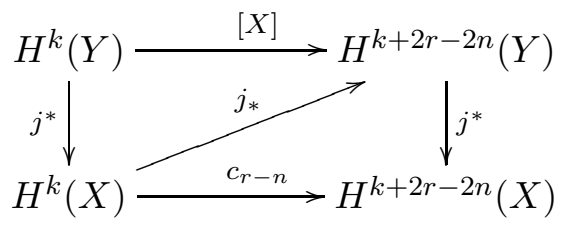


where the horizontal maps are given by the cup-products with the cohomology class $[X] \in H^{2 r-2 n}(Y)$ of $X$ in $Y$, and the top Chern class $c_{r-n}:=c_{r-n}\left(\mathcal{N}_{X / Y}\right)$ of the normal bundle of $X$, respectively. This implies that $j_{*}$ is injective if the cup-product map $c_{r-n} \cup$ is injective. Since the map $j_{*}$ repects the Hodge decompositions on $X$ and $Y$, and since $H^{i}\left(Y, \Omega_{Y}^{j}\right)=0$ for all $0 \leq i, j \leq r$ with $i \neq j$, the vanishing $H^{i}\left(X, \Omega_{X}^{j}\right)=0$ for $i \neq j$, and $j \leq 2 n-r$ follows immediately from the lemma below.

Lemma 12 Let $j: W \rightarrow Z$ be the inclusion of an $n$-dimensional submanifold $W$ into an $r$-dimensional smooth projective variety $Z$, with ample normal bundle. Then the cup-product

$$
H^{k}(W) \stackrel{c_{r-n}}{\longrightarrow} H^{k+2 r-2 n}(W)
$$

with the top Chern class $c_{r-n}:=c_{r-n}\left(\mathcal{N}_{W / Z}\right)$ of the normal bundle of $W$ in $Z$ is injective for $k \leq 2 n-r$.

Proof. Let $\mathcal{N}_{W / Z}$ be the normal bundle of $W$ in $Z$. By assumption, the tautological bundle $\mathcal{O}_{\mathbb{P}\left(\mathcal{N}_{W / Z}\right)}(1)$ on the projective bundle $\pi: \mathbb{P}\left(\mathcal{N}_{W / Z}\right) \rightarrow X$ is ample. Hence we can apply the hard Lefschetz theorem to it. If $h:=$ $c_{1}\left(\mathcal{O}_{\mathbb{P}\left(\mathcal{N}_{W / Z}\right)}(1)\right)$, then we have that for $i \leq \operatorname{dim}\left(\mathbb{P}\left(\mathcal{N}_{W / Z}\right)\right)-1=r-2$ the cup-product map

$$
H^{i}\left(\mathbb{P}\left(\mathcal{N}_{W / Z}\right)\right) \stackrel{h}{\longrightarrow} H^{i+2}\left(\mathbb{P}\left(\mathcal{N}_{W / Z}\right)\right)
$$

is injective. Furthermore we have a relation

$$
0=\sum_{i=0}^{r-n}(-1)^{r-n-i} h^{i} \cdot \pi^{*}\left(c_{r-n-i}\left(\mathcal{N}_{W / Z}\right)\right)
$$

in the cohomology of $\mathbb{P}\left(\mathcal{N}_{W / Z}\right)$. Now let $\alpha \in H^{k}(X)$ and assume $c_{r-n} \cup \alpha=0$. Lifting into the cohomology on $\mathbb{P}\left(\mathcal{N}_{W / Z}\right)$ gives the relation

$$
\pi^{*}\left(c_{r-n} \cup \alpha\right)=\sum_{i=1}^{r-n}(-1)^{i-1} h^{i} \cdot \pi^{*}\left(c_{r-n-i}\left(\mathcal{N}_{W / Z}\right) \cup \alpha\right) .
$$

As mentioned above, if $k \leq 2 n-r$, then the cup-product with $h$ is injective on $H^{k+2 r-2 n-2}\left(\mathbb{P}\left(\mathcal{N}_{W / Z}\right)\right)$ by the Hard Lefschetz theorem. Therefore the 
class $\sum_{i=1}^{r-n}(-1)^{i-1} h^{i-1} \cdot \pi^{*}\left(c_{r-n-i}\left(\mathcal{N}_{W / Z}\right) \cup \alpha\right)$ is equal to 0 as well. But since the expression of a cohomology class on $\mathbb{P}\left(\mathcal{N}_{W / Z}\right)$ by powers of $h$ in this form is unique, we conclude that $\pi^{*}(\alpha)=0$, and hence $\alpha=0$.

Now let us return to the question of how the theorem of Barth-Lefschetz generalizes to smooth projective toric varieties.

Definition 13 Let $X \subset Y$ be $\Delta$-transversal. We say that $X$ has the property (BL) if $\delta_{X, \sigma}=1$ for all $\sigma \in \Delta(t)$ with $2 t \leq 2 n-r$ and $\delta_{X, \sigma} \geq 1$ if $2 t=2 n-r+1$. For such an $X$ in particular $\delta_{X}(t)=\delta(t)$ for all $2 t \leq 2 n-r$.

Remark 14 Note that if $Y=\mathbb{P}^{r}$ we can always assume that $X \subset Y$ of dimension $n$ has the property (BL). This is seen as follows. If $G=P G L(r)$ is the group of homogeneous transformations on $\mathbb{P}^{r}$ then by Kleinman's theorem for any $V(\sigma)$ there is a nonempty open subset $U$ of $G$, such that every transformation of $X$ by an element of $U$ intersects $V(\sigma)$ transversally or not at all. Since there are only finitely many subvarieties $V(\sigma)$ in $Y$ there exists a homogeneous transformation which moves $X$ into $\Delta$-transversal position. Now for $\sigma \in \Delta(t)$ with $2 t \leq 2 n-r+1$ and $X \cap V(\sigma) \neq \emptyset$ put $V:=V(\sigma)$. Since $\operatorname{codim}_{Y} V=t$ we have $\operatorname{codim}_{Y} X \cap V=r-n+t \leq r$, hence $\delta_{X, \sigma} \geq 1$. Let $D_{1}$ and $D_{2}$ be two connected components of $X \cap V$. If $2 t \leq 2 n-r$ then $\operatorname{dim} D_{1}+\operatorname{dim} D_{2}=2(n-t) \geq r$, so $X \cap V$ is connected.

Remark 15 It was conjectured by Hartshorne [ [ $\mathrm{H}]$ that each smooth $X \subset Y$ with ample normal bundle is connected if $2 n-r \geq 0$. This would imply that $\delta_{X, \sigma} \leq 1$ if $\sigma \in \Delta(t)$ with $2 t \leq 2 n-r$. If $X$ is a hypersurface this is always true.

Theorem 16 Let $Y$ be a smooth projective toric variety of dimension $r$ and let $X$ be a n-dimensional submanifold with ample normal bundle which is $\Delta$-transversal. Then the natural map

$$
H^{k}(Y, \mathbb{C}) \rightarrow H^{k}(X, \mathbb{C})
$$

is surjective for $k \leq 2 n-r$ and injective for $k \leq 2 n-r+1$ if and only if $X$ has the property $(B L)$. 
Proof. We use the Hodge decompositions of $H^{k}(Y, \mathbb{C})$ and $H^{k}(X, \mathbb{C})$. Because of proposition 5 it suffices to prove the theorem for the second map of the factorization

$$
H^{i}\left(Y, \Omega_{Y}^{j}\right) \rightarrow H^{i}\left(X, \Omega_{Y}^{j} \mid X\right) \rightarrow H^{i}\left(X, \Omega_{X}^{j}\right)
$$

By theorem 10 it is enough to consider the case $i=j$, since $H^{i}\left(Y, \Omega_{Y}^{j}\right)=0$ if $i \neq j$ and $H^{i}\left(X, \Omega_{Y}^{j} \mid X\right)=0$ if $i \neq j$ and $i+j \leq 2 n-r$. Obviously $H^{0}\left(Y, \mathcal{O}_{Y}\right)=H^{0}\left(X, \mathcal{O}_{X}\right)=\mathbb{C}$ if and only if $\delta_{X}(0)=1$. If $i \geq 1$ we found in the proof of theorem 10 a long exact sequence that computed $H^{i}\left(X, \Omega_{Y}^{i} \mid X\right)$. Using the restriction map from the analogous exact sequence for the toric variety $Y$ to $X$ we get a commutative diagram

$$
\begin{aligned}
& 0 \rightarrow H^{0}\left(Y, \mathcal{K}^{0}(Y ; i)\right) \rightarrow \ldots \rightarrow H^{0}\left(Y, \mathcal{K}^{i}(Y ; i)\right) \rightarrow H^{i}\left(Y, \Omega_{Y}^{i}\right) \rightarrow 0 \\
& \alpha_{i, 0} \downarrow \quad \alpha_{i, i} \downarrow \quad \rho \downarrow \\
& 0 \rightarrow H^{0}\left(\mathcal{K}^{0}(Y ; i) \mid X\right) \rightarrow \ldots \rightarrow H^{0}\left(\mathcal{K}^{i}(Y ; i) \mid X\right) \rightarrow H^{i}\left(\Omega_{Y}^{i} \mid X\right) \rightarrow 0
\end{aligned}
$$

The property $(\mathrm{BL})$ is equivalent to the property that the maps

$$
\alpha_{\nu, k}: H^{0}\left(Y, \mathcal{K}^{k}(Y ; \nu)\right) \rightarrow H^{0}\left(X, \mathcal{K}^{k}(Y ; \nu) \mid X\right)
$$

are isomorphisms if $0 \leq k \leq \frac{2 n-r}{2}$ and injective if $k=\frac{2 n-r+1}{2}$. Note that $\alpha_{\nu, k}$ is injective or an isomorphism for some value of $\nu$ if and only if it is for all possible values of $\nu$. Using this, the theorem follows by an easy induction on $i$.

Remark $17(i)$ Consider the special case $Y=\mathbb{P}^{r}$. For any submanifold $X \subset Y$ the normal bundle is ample. So by what we said in remark 14 this theorem implies the usual Barth-Lefschetz theorem for projective space.

(ii) Because of Kleinman's theorem for homogeneous spaces this result is true for a submanifold $X \subset Y$ with ample normal bundle without any condition on $\Delta$-transversality if $Y$ is equal to a product of projective spaces.

Acknowledgements. I am grateful to the Graduiertenkolleg "Komplexe Mannigfaltigkeiten" at the University of Bayreuth as well as to the University of Bath for their hospitality. In particular I wish to thank Dr. G.K. Sankaran for many helpful conversations. Partially this work has been supported by the EU HCM project "Algebraic Geometry in Europe" (AGE), contract number ERBCHRXCT 940557. 


\section{References}

[B] Barth W., Transplanting cohomology classes in complex-projective space, Amer. J. Math. 92 (1970) 951-967

[BC] Batyrev V., Cox D., On the Hodge structure of projective hypersurfaces in toric varieties, Duke Math. J. 75 No. 2 (1994) 293-338

[D] Debarre O., Fulton-Hansen and Barth-Lefschetz theorems for subvarieties of abelian varieties, Crelle 467 (1995) 187-197

[H] Hartshorne R., Ample Subvarieties of Algebraic Varieties, LNM 156, Springer, Berlin, Heidelberg, New York (1970)

[O] Oda T., Convex Bodies and Algebraic Geometry, Erg. Math. Grenzgeb. 3.Folge, Band 15, Springer, Berlin, Heidelberg, New York (1988)

[LP] Le Potier J., Annulation de la cohomologie à valeurs dans un fibré vectoriel holomorphe positif de rang quelconque, Math. Ann. 218 (1975) $35-53$

[So] Sommese A.J., Complex subspaces of homogeneous complex manifolds II - Homotopy results, Nagoya Math. J. 86 (1982) 101-129

[SZ] Schneider M., Zintl J., The theorem of Barth-Lefschetz as a consequence of Le Potier's vanishing theorem, manuscripta math. 80 (1993) 259-263

Jörg Zintl

Fachbereich Mathematik

Universität Kaiserslautern

Postfach 3049

67653 Kaiserslautern

Germany

zintl@mathematik.uni-kl.de 